\title{
Time-Delay Estimation in Dispersed Spectrum Cognitive Radio Systems
}

\author{
Fatih Kocak, ${ }^{1}$ Hasari Celebi, ${ }^{2}$ Sinan Gezici, ${ }^{1}$ Khalid A. Qaraqe, $^{2}$ \\ Huseyin Arslan, ${ }^{3}$ and H. Vincent Poor ${ }^{4}$ \\ ${ }^{1}$ Department of Electrical and Electronics Engineering, Bilkent University, Bilkent, 06800 Ankara, Turkey \\ ${ }^{2}$ Department of Electrical and Computer Engineering, Texas A\&M University at Qatar, 23874 Doha, Qatar \\ ${ }^{3}$ Department of Electrical Engineering, University of South Florida, 4202 E Fowler Avenue, Tampa, FL 33620, USA \\ ${ }^{4}$ Department of Electrical Engineering, Princeton University, Princeton, NJ 08544, USA
}

Correspondence should be addressed to Fatih Kocak, fkocak@ee.bilkent.edu.tr

Received 28 April 2009; Revised 4 September 2009; Accepted 2 December 2009

Academic Editor: Yonghong Zeng

Copyright ( $) 2010$ Fatih Kocak et al. This is an open access article distributed under the Creative Commons Attribution License, which permits unrestricted use, distribution, and reproduction in any medium, provided the original work is properly cited.

\begin{abstract}
Time-delay estimation is studied for cognitive radio systems, which facilitate opportunistic use of spectral resources. A two-step approach is proposed to obtain accurate time-delay estimates of signals that occupy multiple dispersed bands simultaneously, with significantly lower computational complexity than the optimal maximum likelihood (ML) estimator. In the first step of the proposed approach, an ML estimator is used for each band of the signal in order to estimate the unknown parameters of the signal occupying that band. Then, in the second step, the estimates from the first step are combined in various ways in order to obtain the final time-delay estimate. The combining techniques that are used in the second step are called optimal combining, signal-to-noise ratio (SNR) combining, selection combining, and equal combining. It is shown that the performance of the optimal combining technique gets very close to the Cramer-Rao lower bound at high SNRs. These combining techniques provide various mechanisms for diversity combining for time-delay estimation and extend the concept of diversity in communications systems to the timedelay estimation problem in cognitive radio systems. Simulation results are presented to evaluate the performance of the proposed estimators and to verify the theoretical analysis.
\end{abstract}

\section{Introduction}

Cognitive radio is a promising approach to implement intelligent wireless communications systems [1-8]. Cognitive radios can be perceived as more capable versions of software defined radios in the sense that they have sensing, awareness, learning, adaptation, goal driven autonomous operation, and reconfigurability features $[9,10]$. Thanks to these features, radio resources, such as power and bandwidth, can be used more efficiently [1]. Especially since the electromagnetic spectrum is a precious resource, it must not be wasted. The recent spectrum measurement campaigns in the United States [11] and Europe [12] show that the spectrum is under-utilized; hence, opportunistic use of unoccupied frequency bands is highly desirable.

Cognitive radio provides a solution to the problem of inefficient spectrum utilization by using the vacant frequency spectrum over time in a certain geographical region. In other words, a cognitive radio system can opportunistically use the available spectrum of a legacy system without interfering with the licensed users of that spectrum $[2,3]$. In order to facilitate such opportunistic spectrum utilization, it is important that cognitive radio devices are aware of their positions, and monitor the environment continuously. These location and environmental awareness features of cognitive radios have been studied extensively in the literature $[10,13-$ 19]. In [13], the concept of cognitive radar is introduced, which provides information related to the objects in an environment; that is, it performs environmental sensing. In [14], a radio environment mapping method for cognitive radio networks is studied. Conceptual models for location and environmental awareness engines and cycles are proposed in $[10,15,16]$ for cognitive radio systems. Also, [18] introduces the concept of a topology engine for cognitive 
radios by studying topology information characterization and its applications to cognitive radio networks.

The location awareness feature of cognitive radios can be used in many network optimization applications, such as location-assisted spectrum management, network planning, handover, routing, dynamic channel allocation, and power control $[8,20]$. Location awareness requires that a cognitive radio device performs accurate estimation of its position. One possible way of obtaining position information is to use the Global Positioning System (GPS) technology in cognitive radio systems. However, this is not a very efficient or costeffective solution [17]. As another approach, cognitive radio devices can estimate position-related parameters of signals traveling between them in order to estimate their positions $[17,21]$. Among various position related parameters, the time-delay parameter commonly provides accurate position information with reasonable complexity $[21,22]$. The main focus of this study is time-delay estimation in cognitive radio systems. In other words, the aim is to propose techniques for accurate time-delay estimation in dispersed spectrum systems in order to provide accurate location information to cognitive users. Since the accuracy of location estimation increases as the accuracy of time-delay estimation increases, design of time-delay estimators with high accuracy and reasonable complexity is crucial for the location awareness feature of a cognitive radio system [21].

Time-delay estimation in cognitive radio systems differs from conventional time-delay estimation mainly due to the fact that a cognitive radio system can transmit and receive over multiple dispersed bands. In other words, since a cognitive radio device can utilize the spectral holes of a legacy system, it can have a spectrum that consists of multiple bands that are dispersed over a wide range of frequencies (cf. Figure 1). In [23], the theoretical limits on time-delay estimation are studied for dispersed spectrum cognitive radio systems, and the effects of carrier frequency offset (CFO) and modulation schemes of training signals on the accuracy of time-delay estimation are quantified. The expressions for the theoretical limits indicate that frequency diversity can be utilized in time-delay estimation. Similarly, the effects of spatial diversity on time-delay estimation are studied in [24] for single-input multiple-output (SIMO) systems. In addition, the effects of multiple antennas on time-delay estimation and synchronization problems are investigated in [25].

In this paper, time-delay estimation is studied for dispersed spectrum cognitive radio systems. First, it is observed that maximum likelihood (ML) estimation is not very practical for time-delay estimation in such systems. Then, a two-step time-delay estimation approach is proposed in order to provide accurate time-delay estimation with significantly lower computational complexity than that of the optimal ML estimator. In the proposed scheme, the receiver consists of multiple branches and each branch processes the part of the received signal that occupies the corresponding frequency band. An ML estimator is used in each branch in order to estimate the unknown parameters of the signal observed in that branch. Then, in the second step, the estimates from all the branches are combined to obtain the

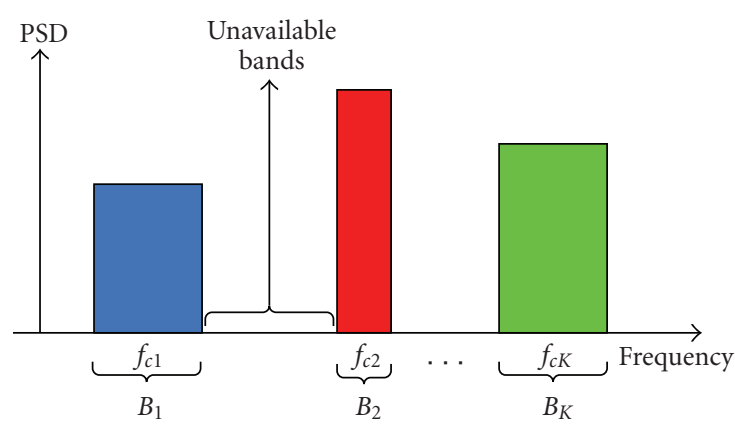

FIGURE 1: Illustration of dispersed spectrum utilization in cognitive radio systems.

final time-delay estimate. Various techniques are proposed for the combining operation in the second step: Optimal combining, signal-to-noise ratio (SNR) combining, selection combining, and equal combining. The biases and variances of the time-delay estimators that employ these combining techniques are investigated. It is shown that the optimal combining technique results in a mean-squared error (MSE) that approximates the Cramer-Rao lower bound (CRLB) at high SNRs. Simulation results are provided in order to compare the performance of the proposed time-delay estimators. In a more generic perspective, this study focuses on the utilization of frequency diversity for a parameter estimation problem. Therefore, the proposed estimators can be applied to other systems that have frequency diversity as well.

The remainder of the paper is organized as follows. In Section 2, the signal model is introduced and the signal at each branch of the receiver is described. In Section 3, the optimal ML receiver is obtained, and the CRLBs on time-delay estimation in dispersed spectrum cognitive radio systems are described. The proposed two-step timedelay estimation approach is studied in Section 4 . Then, in Section 5 , the optimality properties of the proposed timedelay estimators are investigated. Finally, simulation results are presented in Section 6, and concluding remarks are made in Section 7.

\section{Signal Model}

A cognitive radio system that occupies $K$ dispersed frequency bands is considered as shown in Figure 1. The transmitter sends a signal occupying all the $K$ bands simultaneously, and the receiver aims to calculate the time-delay of the incoming signal.

One approach for designing such a system involves the use of orthogonal frequency division multiplexing (OFDM). In this approach, the received signal is considered as a single OFDM signal with zero coefficients at the subcarriers corresponding to the unavailable bands [26-28]. Then, the signal can be processed as in conventional OFDM receivers. The main drawback of this approach is that it requires processing of very large bandwidths when the available spectrum is dispersed over a wide range of frequencies. 


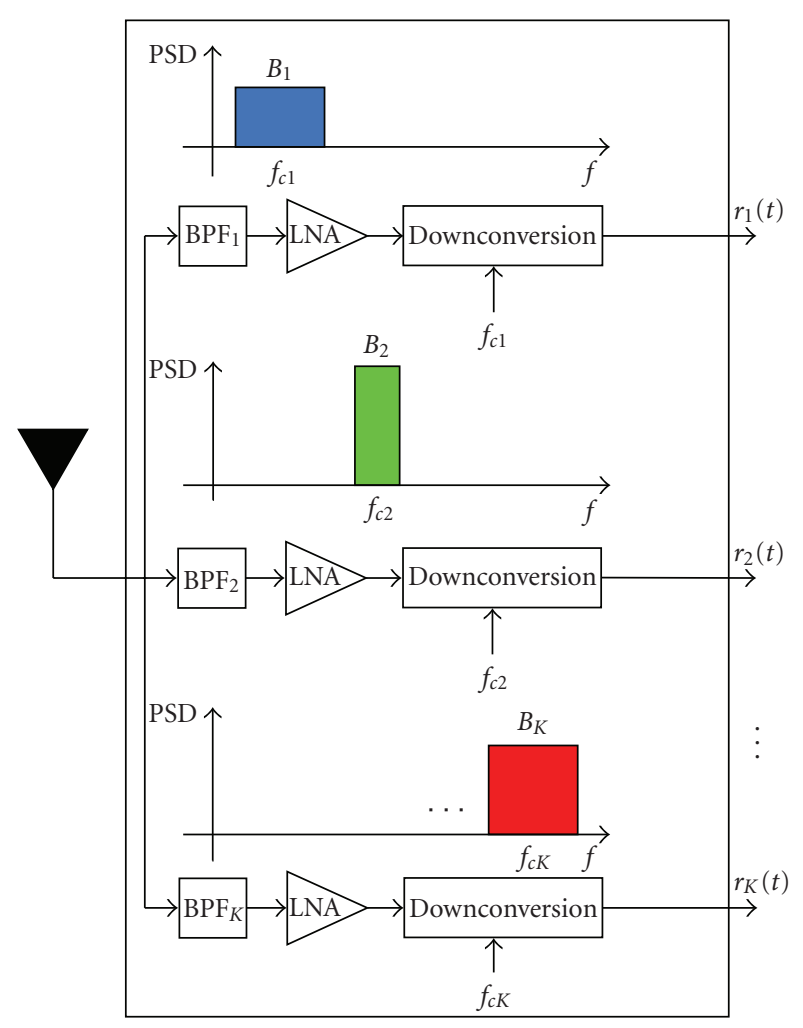

FIGURE 2: Block diagram of the front-end of a cognitive radio receiver, where BPF and LNA refer to band-pass filter and low-noise amplifier, respectively.

Therefore, the design of RF components, such as filters and low-noise amplifiers (LNAs), can become very complex and costly, and result in components with high power consumption [29]. In such scenarios, it can be more practical to process the received signal in multiple branches, as shown in Figure 2. In that case, each branch processes one available band and down-converts the signal according to the center frequency of that band. Therefore, signals with narrower bandwidths can be processed at each branch.

For the receiver model in Figure 2, the baseband representation of the received signal in the $i$ th branch can be modeled as

$$
r_{i}(t)=\alpha_{i} \mathrm{e}^{j \omega_{i} t} s_{i}(t-\tau)+n_{i}(t),
$$

for $i=1, \ldots, K$, where $\tau$ is the time-delay of the signal, $\alpha_{i}=$ $a_{i} \mathrm{e}^{j \phi_{i}}$ and $\omega_{i}$ represent, respectively, the channel coefficient and the CFO for the signal in the $i$ th branch, $s_{i}(t)$ is the baseband representation of the transmitted signal in the $i$ th band, and $n_{i}(t)$ is modeled as complex white Gaussian noise with independent components, each having spectral density $\sigma_{i}^{2}$.

The signal model in (1) assumes that the signal in each branch can be modeled as a narrowband signal. Hence, a single complex channel coefficient is used to represent the fading of each signal.

The system model considered in this study falls within the framework of cognitive radio systems, since the cognitive user first needs to detect the available frequency bands, and then to adapt its receiver parameters accordingly. Therefore, the spectrum sensing and adaptation features of cognitive systems are assumed for the considered system in this study $[9,10]$.

\section{Optimal Time-Delay Estimation and Theoretical Limits}

Accurate estimation of the time-delay parameter $\tau$ in (1) is quite challenging due to the presence of unknown channel coefficients and CFOs. For a system with $K$ bands, there are $3 K$ nuisance parameters. In other words, the vector $\boldsymbol{\theta}$ of unknown parameters can be expressed as

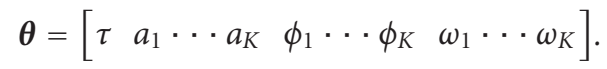

When the signals in (1) are observed over the interval $[0, T]$, the log-likelihood function for $\boldsymbol{\theta}$ is given by [30]

$$
\Lambda(\boldsymbol{\theta})=c-\sum_{i=1}^{K} \frac{1}{2 \sigma_{i}^{2}} \int_{0}^{T}\left|r_{i}(t)-\alpha_{i} \mathrm{e}^{j \omega_{i} t} s_{i}(t-\tau)\right|^{2} \mathrm{~d} t,
$$

where $c$ is a constant that is independent of $\boldsymbol{\theta}$ (the unknown parameters are assumed to be constant during the observation interval). Then, the ML estimate for $\boldsymbol{\theta}$ can be obtained from (3) as [23]

$\widehat{\boldsymbol{\theta}}_{\mathrm{ML}}$

$=\arg \max _{\boldsymbol{\theta}}\left\{\sum_{i=1}^{K} \frac{1}{\sigma_{i}^{2}} \int_{0}^{T} \mathcal{R}\left\{\alpha_{i}^{*} \mathrm{e}^{-j \omega_{i} t} r_{i}(t) s_{i}^{*}(t-\tau)\right\} \mathrm{d} t-\sum_{i=1}^{K} \frac{E_{i}\left|\alpha_{i}\right|^{2}}{2 \sigma_{i}^{2}}\right\}$,

where $E_{i}=\int_{0}^{T}\left|s_{i}(t-\tau)\right|^{2} \mathrm{~d} t$ is the signal energy, and $\mathcal{R}$ represents the operator that selects the real part of its argument.

It is observed from (4) that the ML estimator requires an optimization over a $(3 K+1)$-dimensional space, which is quite challenging in general. Therefore, the aim of this study is to propose low-complexity time-delay estimation algorithms with comparable performance to that of the ML estimator in (4). In other words, accurate time-delay estimation algorithms are studied under practical constraints on the processing power of the receiver. Since the ML estimator is difficult to implement, the performance comparisons will be made with respect to the theoretical limits on time-delay estimation (an ML estimator achieves the CRLB asymptotically under certain conditions [30]). In [23], the CRLBs on the MSEs of unbiased time-delay estimators are obtained for the signal model in (1). When the baseband representation of the signals in different branches is of the form $s_{i}(t)=\sum_{l} d_{i, l} p_{i}\left(t-l T_{i}\right)$, where $d_{i, l}$ denotes the complex training data and $p_{i}(t)$ is a pulse with duration $T_{i}$, the CRLB is expressed as

$$
\mathrm{E}\left\{(\hat{\tau}-\tau)^{2}\right\} \geq\left(\sum_{i=1}^{K} \frac{a_{i}^{2}}{\sigma_{i}^{2}}\left(\widetilde{E}_{i}-\frac{\left(\hat{E}_{i}^{\mathrm{R}}\right)^{2}}{E_{i}}\right)\right)^{-1},
$$




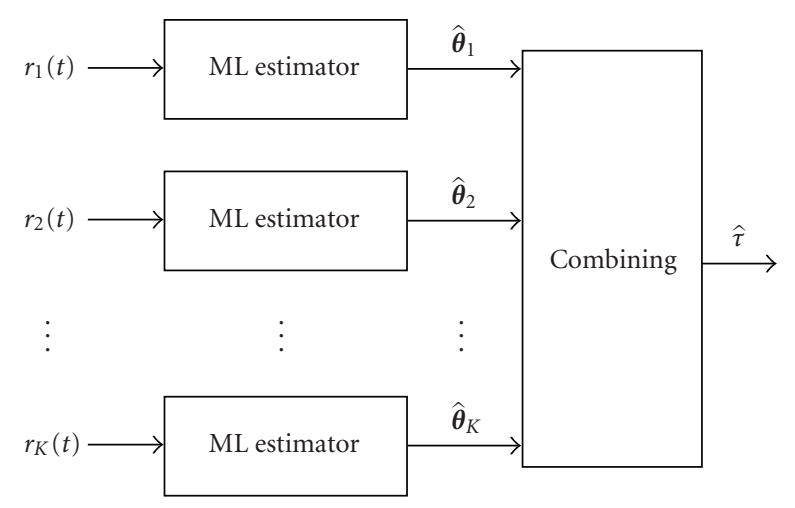

FIgURE 3: The block diagram of the proposed time-delay estimation approach. The signals $r_{1}(t), \ldots, r_{K}(t)$ are obtained at the front-end of the receiver as shown in Figure 2.

where

$$
\begin{gathered}
\widetilde{E}_{i}=\int_{0}^{T}\left|s_{i}^{\prime}(t-\tau)\right|^{2} \mathrm{~d} t, \\
\widehat{E}_{i}^{\mathrm{R}}=\int_{0}^{T} \mathcal{R}\left\{s_{i}^{\prime}(t-\tau) s_{i}^{*}(t-\tau)\right\} \mathrm{d} t,
\end{gathered}
$$

with $s^{\prime}(t)$ representing the first derivative of $s(t)$. In the special case of $\left|d_{i, l}\right|=\left|d_{i}\right|$ for all $l$ and $p_{i}(t)$ satisfying $p_{i}(0)=p_{i}\left(T_{i}\right)$ for $i=1, \ldots, K,(5)$ becomes [23]

$$
\mathrm{E}\left\{(\hat{\tau}-\tau)^{2}\right\} \geq\left(\sum_{i=1}^{K} \frac{\widetilde{E}_{i} a_{i}^{2}}{\sigma_{i}^{2}}\right)^{-1} .
$$

It is observed from (5) and (8) that frequency diversity can be useful in time-delay estimation. For example, when one of the bands is in a deep fade (i.e., small $a_{i}^{2}$ ), some other bands can still be in good condition to facilitate accurate time-delay estimation.

\section{Two-Step Time-Delay Estimation and Diversity Combining}

Due to the complexity of the ML estimator in (4), a two-step time-delay estimation approach is proposed in this paper, as shown in Figure 3. Two-step approaches are commonly used in optimization/estimation problems in order to provide suboptimal solutions with reduced computational complexity $[31,32]$. In the proposed estimator, each branch of the receiver performs estimation of the time-delay, the channel coefficient, and the CFO related to the signal in that branch. Then, the estimates from all the branches are used to obtain the final time-delay estimate as shown in Figure 3. In the following sections, the details of the proposed approach are explained, and the utilization of frequency diversity in timedelay estimation is explained.

4.1. First Step: Parameter Estimation at Different Branches. In the first step of the proposed approach, the unknown parameters of each received signal are estimated at the corresponding receiver branch according to the ML criterion (cf. Figure 3). Based on the signal model in (1), the likelihood function at branch $i$ can be expressed as

$$
\Lambda_{i}\left(\boldsymbol{\theta}_{i}\right)=c_{i}-\frac{1}{2 \sigma_{i}^{2}} \int_{0}^{T}\left|r_{i}(t)-\alpha_{i} \mathrm{e}^{j \omega_{i} t} s_{i}(t-\tau)\right|^{2} \mathrm{~d} t,
$$

for $i=1, \ldots, K$, where $\boldsymbol{\theta}_{i}=\left[\begin{array}{lll}\tau & a_{i} \phi_{i} & \omega_{i}\end{array}\right]$ represents the vector of unknown parameters related to the signal at the $i$ th branch, $r_{i}(t)$, and $c_{i}$ is a constant that is independent of $\boldsymbol{\theta}_{i}$.

From (9), the ML estimator at branch $i$ can be stated as

$$
\widehat{\boldsymbol{\theta}}_{i}=\arg \min _{\boldsymbol{\theta}_{i}} \int_{0}^{T}\left|r_{i}(t)-\alpha_{i} \mathrm{e}^{j \omega_{i} t} s_{i}(t-\tau)\right|^{2} \mathrm{~d} t,
$$

where $\hat{\boldsymbol{\theta}}_{i}=\left[\hat{\tau}_{i} \hat{a}_{i} \hat{\phi}_{i} \hat{\omega}_{i}\right]$ is the vector of estimates at the $i$ th branch. After some manipulation, the solution of (10) can be obtained as

$$
\begin{aligned}
& {\left[\hat{\tau}_{i} \hat{\phi}_{i} \hat{\omega}_{i}\right]=\arg \max _{\phi_{i}, \omega_{i}, \tau_{i}}\left|\int_{0}^{T} \mathcal{R}\left\{r_{i}(t) \mathrm{e}^{-j\left(\omega_{i} t+\phi_{i}\right)} s_{i}^{*}\left(t-\tau_{i}\right)\right\} \mathrm{d} t\right|,} \\
& \hat{a}_{i}=\frac{1}{E_{i}} \int_{0}^{T} \mathcal{R}\left\{r_{i}(t) \mathrm{e}^{-j\left(\hat{\omega}_{i} t+\hat{\phi}_{i}\right)} s_{i}^{*}\left(t-\hat{\tau}_{i}\right)\right\} \mathrm{d} t .
\end{aligned}
$$

In other words, at each branch, optimization over a threedimensional space is required to obtain the unknown parameters. Compared to the ML estimator in Section 3, the optimization problem in $(4)$ over $(3 K+1)$ variables is reduced to $K$ optimization problems over three variables, which results in a significant amount of reduction in the computational complexity.

In the absence of CFO; that is, $\omega_{i}=0$ for all $i,(11)$ and (12) reduce to

$$
\begin{gathered}
{\left[\hat{\tau}_{i} \hat{\phi}_{i}\right]=\arg \max _{\phi_{i}, \tau_{i}}\left|\int_{0}^{T} \mathcal{R}\left\{r_{i}(t) \mathrm{e}^{-j \phi_{i}} s_{i}^{*}\left(t-\tau_{i}\right)\right\} \mathrm{d} t\right|,} \\
\hat{a}_{i}=\frac{1}{E_{i}} \int_{0}^{T} \mathcal{R}\left\{r_{i}(t) \mathrm{e}^{-j \hat{\phi}_{i}} s_{i}^{*}\left(t-\hat{\tau}_{i}\right)\right\} \mathrm{d} t .
\end{gathered}
$$

In that case, the optimization problem at each branch is performed over only two dimensions. This scenario is valid when the carrier frequency of each band is known accurately.

4.2. Second Step: Combining Estimates from Different Branches. After obtaining $K$ different time-delay estimates, $\widehat{\tau}_{1}, \ldots, \hat{\tau}_{K}$, in (11), the second step combines those estimates according to one of the criteria below and makes the final time-delay estimate (cf. Figure 3).

4.2.1. Optimal Combining. According to the "optimal" combining criterion (the optimality properties of this combining technique are investigated in Section 5), the time-delay estimate is obtained as

$$
\hat{\tau}=\frac{\sum_{i=1}^{K} \kappa_{i} \hat{\tau}_{i}}{\sum_{i=1}^{K} \kappa_{i}}
$$


where $\hat{\tau}_{i}$ is the time-delay estimate of the $i$ th branch, which is obtained from (11), and

$$
\kappa_{i}=\frac{\hat{a}_{i}^{2} \widetilde{E}_{i}}{\sigma_{i}^{2}},
$$

with $\widetilde{E}_{i}$ being defined in (6). In other words, the optimal combining technique estimates the time-delay as a weighted average of the time-delays of different branches, where the weights are chosen as proportional to the multiplication of the SNR estimate, $E_{i} \hat{a}_{i}^{2} / \sigma_{i}^{2}$, and $\widetilde{E}_{i} / E_{i}$. Since $\widetilde{E}_{i}$ is defined as the energy of the first derivative of $s_{i}(t)$ as in $(6), \widetilde{E}_{i} / E_{i}$ can be expressed, using Parseval's relation, as $\widetilde{E}_{i} / E_{i}=4 \pi^{2} \beta_{i}^{2}$, where $\beta_{i}$ is the effective bandwidth of $s_{i}(t)$, which is defined as [30]

$$
\beta_{i}^{2}=\frac{1}{E_{i}} \int_{-\infty}^{\infty} f^{2}\left|S_{i}(f)\right|^{2} \mathrm{~d} f
$$

with $S_{i}(f)$ denoting the Fourier transform of $s_{i}(t)$. Therefore, it is concluded that the optimal combining technique assigns a weight to the time-delay estimate of a given branch in proportion to the product of the SNR estimate and the effective bandwidth related to that branch. The intuition behind this combining technique is the fact that signals with larger effective bandwidths and/or larger SNRs facilitate more accurate time-delay estimation [30]; hence, their weights should be larger in the combining process. This intuition is verified theoretically in Section 5 .

4.2.2. SNR Combining. The second technique combines the time-delay estimates in the first step according to the SNR estimates at the respective branches. In other words, the time-delay estimate is obtained as

$$
\hat{\tau}=\frac{\sum_{i=1}^{K} \gamma_{i} \hat{\tau}_{i}}{\sum_{i=1}^{K} \gamma_{i}}
$$

where

$$
\gamma_{i}=\frac{\hat{a}_{i}^{2} E_{i}}{\sigma_{i}^{2}} .
$$

Note that $\gamma_{i}$ defines the SNR estimate at branch $i$. In other words, this technique considers only the SNR estimates at the branches in order to determine the combining coefficients, and does not take the signal bandwidths into account.

It is observed from (15)-(19) that the optimal combining and the SNR combining techniques become equivalent if $\widetilde{E}_{1} / E_{1}=\cdots=\widetilde{E}_{K} / E_{K}$. Since $\widetilde{E}_{i} / E_{i}=4 \pi^{2} \beta_{i}^{2}$, where $\beta_{i}$ is the effective bandwidth defined in (17), the two techniques are equivalent when the effective bandwidths of the signals at different branches are all equal.

4.2.3. Selection Combining-1 (SC-1). Another technique for obtaining the final time-delay estimate is to determine the "best" branch and to use its estimate as the final time-delay estimate. According to SC- 1 , the best branch is defined as the one that has the maximum value of $\kappa_{i}=$ $\hat{a}_{i}^{2} \widetilde{E}_{i} / \sigma_{i}^{2}$, for $i=1, \ldots, K$. In other words, the branch with the maximum multiplication of the SNR estimate and the effective bandwidth is determined as the best branch and its estimate is used as the final one. That is,

$$
\hat{\tau}=\hat{\tau}_{m}, \quad m=\arg \max _{i \in\{1, \ldots, K\}}\left\{\frac{\hat{a}_{i}^{2} \widetilde{E}_{i}}{\sigma_{i}^{2}}\right\},
$$

where $\hat{\tau}_{m}$ represents the time-delay estimate at the $m$ th branch.

4.2.4. Selection Combining-2 (SC-2). Similar to SC-1, SC2 selects the "best" branch and uses its estimate as the final time-delay estimate. However, according to SC-2, the best branch is defined as the one with the maximum SNR. Therefore, the time-delay estimate is obtained as follows according to SC-2:

$$
\hat{\tau}=\hat{\tau}_{m}, \quad m=\arg \max _{i \in\{1, \ldots, K\}}\left\{\frac{\hat{a}_{i}^{2} E_{i}}{\sigma_{i}^{2}}\right\},
$$

where $\hat{\tau}_{m}$ represents the time-delay estimate at the $m$ th branch.

SC-1 and SC-2 become equivalent when the effective bandwidths of the signals at different branches are all equal.

4.2.5. Equal Combining. The equal combining technique assigns equal weights to the estimates from different branches and obtains the time-delay estimate as follows:

$$
\hat{\tau}=\frac{1}{K} \sum_{i=1}^{K} \widehat{\tau}_{i}
$$

Considering the proposed combining techniques above, it is observed that they are similar to diversity combining techniques in communications systems [33]. However, the main difference is the following. The aim is to maximize the SNR or to reduce the probability of symbol error in communications systems [33]; whereas, in the current problem, it is to reduce the MSE of time-delay estimation. In other words, this study considers diversity combining for time-delay estimation, where the diversity results from the dispersed spectrum utilization of the cognitive radio system.

\section{On the Optimality of Two-Step Time-delay Estimation}

In this section, the asymptotic optimality properties of the two-step time-delay estimators proposed in the previous section are investigated. In order to analyze the performance of the estimators at high SNRs, the result in [24] for timedelay estimation at multiple receive antennas is extended to the scenario in this paper.

Lemma 1. Consider any linear modulation of the form $s_{i}(t)=$ $\sum_{l} d_{i, l} p_{i}\left(t-l T_{i}\right)$, where $d_{i, l}$ denotes the complex data for the lth symbol of signal $i$, and $p_{i}(t)$ represents a pulse with duration $T_{i}$. Assume that $\int_{-\infty}^{\infty} s_{i}^{\prime}(t-\tau) s_{i}^{*}(t-\tau) \mathrm{d} t=0$, for $i=1, \ldots, K$, then, for the signal model in (1), the delay estimate in (11) and 
the channel amplitude estimate in (12) can be modeled, at high SNR, as

$$
\begin{gathered}
\hat{\tau}_{i}=\tau+v_{i}, \\
\hat{a}_{i}=a_{i}+\eta_{i},
\end{gathered}
$$

for $i=1, \ldots, K$, where $\nu_{i}$ and $\eta_{i}$ are independent zero mean Gaussian random variables with variances $\sigma_{i}^{2} /\left(\widetilde{E}_{i} a_{i}^{2}\right)$ and $\sigma_{i}^{2} / E_{i}$, respectively. In addition, $\nu_{i}$ and $\nu_{j}\left(\eta_{i}\right.$ and $\left.\eta_{j}\right)$ are independent for $i \neq j$.

Proof. The proof uses the derivations in [23] in order to extend Lemma 1 in [24] to the cases with CFO. At high SNRs, the ML estimate $\hat{\boldsymbol{\theta}}_{i}$ of $\boldsymbol{\theta}_{i}=\left[\begin{array}{lll}\tau & a_{i} & \phi_{i} \\ \omega_{i}\end{array}\right]$ in (11) and (12) is approximately distributed as a jointly Gaussian random variable with the mean being equal to $\boldsymbol{\theta}_{i}$ and the covariance matrix being given by the inverse of the Fisher information matrix (FIM) for observation $r_{i}(t)$ in (1) over $[0, T]$. Then, the results in [23] can be used to show that, under the conditions in the lemma, the first $2 \times 2$ block of the covariance matrix can be obtained as $\operatorname{diag}\left\{\sigma_{i}^{2} /\left(\widetilde{E}_{i} a_{i}^{2}\right), \sigma_{i}^{2} / E_{i}\right\}$. Therefore, $\hat{\tau}_{i}$ and $\hat{a}_{i}$ can be modeled as in (23) and (24). In addition, since the noise components at different branches are independent, the estimates are independent for different branches.

Based on Lemma 1, the asymptotic unbiasedness properties of the estimators in Section 4 can be verified. First, it is observed from Lemma 1 that $\mathrm{E}\left\{\hat{\tau}_{i}\right\}=\tau$. Considering the optimal combining technique in (15) as an example, the unbiasedness property can be shown as

$$
\begin{aligned}
\mathrm{E}\left\{\hat{\tau} \mid \hat{a}_{1}, \ldots, \hat{a}_{K}\right\} & =\frac{\sum_{i=1}^{K} \kappa_{i} \mathrm{E}\left\{\hat{\tau}_{i} \mid \hat{a}_{1}, \ldots, \hat{a}_{K}\right\}}{\sum_{i=1}^{K} \kappa_{i}} \\
& =\frac{\sum_{i=1}^{K} \kappa_{i} \mathrm{E}\left\{\hat{\tau}_{i} \mid \hat{a}_{i}\right\}}{\sum_{i=1}^{K} \kappa_{i}}=\tau,
\end{aligned}
$$

where $\kappa_{i}=\hat{a}_{i}^{2} \widetilde{E}_{i} / \sigma_{i}^{2}$. Since $\mathrm{E}\left\{\hat{\tau} \mid \hat{a}_{1}, \ldots, \hat{a}_{K}\right\}$ does not depend on $\hat{a}_{1}, \ldots, \hat{a}_{K}, \mathrm{E}\{\hat{\tau}\}=\mathrm{E}\left\{\mathrm{E}\left\{\hat{\tau} \mid \hat{a}_{1}, \ldots, \hat{a}_{K}\right\}\right\}=\tau$. In other words, since for each specific value of $\hat{a}_{i}, \hat{\tau}_{i}$ is unbiased $(i=1, \ldots, K)$, the weighted average of $\hat{\tau}_{1}, \ldots, \hat{\tau}_{K}$ is also unbiased. Similar arguments can be used to show that all the two-step estimators described in Section 4 are asymptotically unbiased.

Regarding the variance of the estimators, it can be shown that the optimal combining technique has a variance that is approximately equal to the CRLB at high SNRs (in fact, this is the main reason why this combining technique is called optimal). To that aim, the conditional variance of $\hat{\tau}$ in (15) given $\hat{a}_{1}, \ldots, \hat{a}_{K}$ is obtained as follows:

$$
\operatorname{Var}\left\{\hat{\tau} \mid \hat{a}_{1}, \ldots, \hat{a}_{K}\right\}=\frac{\sum_{i=1}^{K} \kappa_{i}^{2} \operatorname{Var}\left\{\hat{\tau}_{i} \mid \hat{a}_{1}, \ldots, \hat{a}_{K}\right\}}{\left(\sum_{i=1}^{K} \kappa_{i}\right)^{2}}
$$

where the independence of the time-delay estimates is used to obtain the result (cf. Lemma 1). Since $\operatorname{Var}\left\{\hat{\tau}_{i} \mid \hat{a}_{1}, \ldots, \hat{a}_{K}\right\}=$ $\operatorname{Var}\left\{\hat{\tau}_{i} \mid \hat{a}_{i}\right\}=\sigma_{i}^{2} /\left(\widetilde{E}_{i} a_{i}^{2}\right)$ from Lemma 1 and $\kappa_{i}=\hat{a}_{i}^{2} \widetilde{E}_{i} / \sigma_{i}^{2}$, (26) can be expressed as

$$
\begin{aligned}
\operatorname{Var}\left\{\hat{\tau} \mid \hat{a}_{1}, \ldots, \hat{a}_{K}\right\} & =\frac{\sum_{i=1}^{K}\left(\hat{a}_{i}^{4} \widetilde{E}_{i}^{2} / \sigma_{i}^{4}\right)\left(\sigma_{i}^{2} /\left(\widetilde{E}_{i} a_{i}^{2}\right)\right)}{\left(\sum_{i=1}^{K}\left(\hat{a}_{i}^{2} \widetilde{E}_{i} / \sigma_{i}^{2}\right)\right)^{2}} \\
& =\sum_{i=1}^{K} \frac{\hat{a}_{i}^{4} \widetilde{E}_{i}}{a_{i}^{2} \sigma_{i}^{2}}\left(\sum_{i=1}^{K} \frac{\hat{a}_{i}^{2} \widetilde{E}_{i}}{\sigma_{i}^{2}}\right)^{-2} .
\end{aligned}
$$

Lemma 1 states that at high SNRs, $\hat{a}_{i}$ is distributed as a Gaussian random variable with mean $a_{i}$ and variance $\sigma_{i}^{2} / E_{i}$. Therefore, for sufficiently large values of $E_{i} / \sigma_{i}^{2}, \ldots, E_{K} / \sigma_{K}^{2}$, (27) can be approximated by

$$
\operatorname{Var}\left\{\hat{\tau} \mid \hat{a}_{1}, \ldots, \hat{a}_{K}\right\} \approx\left(\sum_{i=1}^{K} \frac{\widetilde{E}_{i} a_{i}^{2}}{\sigma_{i}^{2}}\right)^{-1},
$$

which is equal to CRLB expression in (8). Therefore, the optimal combining technique in (15) results in an approximately optimal estimator at high SNRs.

The variances of the other combining techniques in Section 4 can be obtained in a straightforward manner and it can be shown that the asymptotic variances are larger than the CRLB in general. For example, for the SNR combining technique in (18), the conditional variance can be calculated as

$$
\begin{aligned}
\operatorname{Var}\left\{\hat{\tau} \mid \hat{a}_{1}, \ldots, \hat{a}_{K}\right\} & =\frac{\sum_{i=1}^{K}\left(\hat{a}_{i}^{4} E_{i}^{2} / \sigma_{i}^{4}\right)\left(\sigma_{i}^{2} /\left(\widetilde{E}_{i} a_{i}^{2}\right)\right)}{\left(\sum_{i=1}^{K}\left(\hat{a}_{i}^{2} E_{i} / \sigma_{i}^{2}\right)\right)^{2}} \\
& =\sum_{i=1}^{K} \frac{\hat{a}_{i}^{4} E_{i}^{2}}{a_{i}^{2} \widetilde{E}_{i} \sigma_{i}^{2}}\left(\sum_{i=1}^{K} \frac{\hat{a}_{i}^{2} E_{i}}{\sigma_{i}^{2}}\right)^{-2},
\end{aligned}
$$

which, for sufficiently large SNRs, becomes

$$
\operatorname{Var}\left\{\hat{\tau} \mid \hat{a}_{1}, \ldots, \hat{a}_{K}\right\} \approx \sum_{i=1}^{K} \frac{a_{i}^{2} E_{i}^{2}}{\widetilde{E}_{i} \sigma_{i}^{2}}\left(\sum_{i=1}^{K} \frac{a_{i}^{2} E_{i}}{\sigma_{i}^{2}}\right)^{-2} .
$$

Then, from the Cauchy-Schwarz inequality, the following condition is obtained:

$$
\begin{aligned}
\operatorname{Var}\left\{\hat{\tau} \mid \hat{a}_{1}, \ldots, \hat{a}_{K}\right\} & \approx \frac{\sum_{i=1}^{K}\left(a_{i}^{2} E_{i}^{2} /\left(\widetilde{E}_{i} \sigma_{i}^{2}\right)\right)}{\left(\sum_{i=1}^{K}\left(a_{i} E_{i} /\left(\sigma_{i} \sqrt{\widetilde{E}_{i}}\right)\right)\left(a_{i} \sqrt{\widetilde{E}_{i}} / \sigma_{i}\right)\right)^{2}} \\
& \geq \frac{\sum_{i=1}^{K}\left(a_{i}^{2} E_{i}^{2} /\left(\widetilde{E}_{i} \sigma_{i}^{2}\right)\right)}{\sum_{i=1}^{K}\left(a_{i}^{2} E_{i}^{2} /\left(\widetilde{E}_{i} \sigma_{i}^{2}\right)\right) \sum_{i=1}^{K}\left(a_{i}^{2} \widetilde{E}_{i} / \sigma_{i}^{2}\right)} \\
& =\mathrm{CRLB},
\end{aligned}
$$

which holds with equality if and only if $\widetilde{E}_{1} / E_{1}=\cdots=$ $\widetilde{E}_{K} / E_{K}$ (or, $\beta_{1}=\cdots=\beta_{K}$ ). In fact, under that condition, 
the optimal combining and the SNR combining techniques become identical as mentioned in Section 4, since $\kappa_{i}=$ $\hat{a}_{i}^{2} \widetilde{E}_{i} / \sigma_{i}^{2}=\left(\widetilde{E}_{i} / E_{i}\right)\left(E_{i} \hat{a}_{i}^{2} / \sigma_{i}^{2}\right)=\left(\widetilde{E}_{i} / E_{i}\right) \gamma_{i}$ (cf. (16) and (19)). In other words, when the effective bandwidths of the signals at different branches are not equal, the asymptotic variance of the SNR combining technique is strictly larger than the CRLB.

Regarding the selection combining approaches in (20) and (21), similar conclusions as for the diversity combining techniques in communications systems can be made [33]. Specifically, SC-1 and SC-2 perform worse than the optimal combining and the SNR combining techniques, respectively, in general. However, when the estimate of a branch is significantly more accurate than the others, the performance of the selection combining approach can get very close to the optimal combining or the SNR combining technique. However, when the branches have similar estimation accuracies, the selection combining techniques can perform significantly worse. The conditional variances of the selection combining techniques can be approximated at high SNR as

$$
\operatorname{Var}\left\{\hat{\tau} \mid \hat{a}_{1}, \ldots, \hat{a}_{K}\right\} \approx \min \left\{\frac{\sigma_{1}^{2}}{\widetilde{E}_{1} a_{1}^{2}}, \ldots, \frac{\sigma_{K}^{2}}{\widetilde{E}_{K} a_{K}^{2}}\right\},
$$

for SC-1, and

$$
\operatorname{Var}\left\{\hat{\tau} \mid \hat{a}_{1}, \ldots, \hat{a}_{K}\right\} \approx \frac{E_{m}}{\widetilde{E}_{m}} \min \left\{\frac{\sigma_{1}^{2}}{E_{1} a_{1}^{2}}, \ldots, \frac{\sigma_{K}^{2}}{E_{K} a_{K}^{2}}\right\},
$$

for SC-2, where $m=\arg \min _{i \in\{1, \ldots, K\}}\left\{\sigma_{i}^{2} /\left(E_{i} \hat{a}_{i}^{2}\right)\right\}$. From (32) and (33), it is observed that if $\widetilde{E}_{1} / E_{1}=\cdots=\widetilde{E}_{K} / E_{K}\left(\beta_{1}=\right.$ $\left.\cdots=\beta_{K}\right)$, then the asymptotic variances of the SC- 1 and SC-2 techniques become equivalent.

Finally, for the equal combining technique, the variance can be obtained from (22) as

$$
\operatorname{Var}\{\hat{\tau}\}=\frac{1}{K^{2}} \sum_{i=1}^{K} \frac{\sigma_{i}^{2}}{\widetilde{E}_{i} a_{i}^{2}}
$$

In general, the equal combining technique is expected to have the worst performance since it does not make use of any information about the SNR or the signal bandwidths in the estimation of the time-delay.

\section{Simulation Results}

In this section, simulations are performed in order to evaluate the performance of the proposed time-delay estimators and compare them with each other and against the CRLBs. The signal $s_{i}(t)$ in (1) corresponding to each branch is modeled by the Gaussian doublet given by

$$
s_{i}(t)=A_{i}\left(1-\frac{4 \pi\left(t-1.25 \zeta_{i}\right)^{2}}{\zeta_{i}^{2}}\right) e^{-2 \pi\left(t-1.25 \zeta_{i}\right)^{2} / \zeta_{i}^{2}},
$$

where $A_{i}$ and $\zeta_{i}$ are the parameters that are used to adjust the pulse energy and the pulse width, respectively. The bandwidth of $s_{i}(t)$ in (35) can approximately be expressed as $B_{i} \approx 1 /\left(2.5 \zeta_{i}\right)$ [29]. For the following simulations, $A_{i}$ values are adjusted to generate unit-energy pulses.

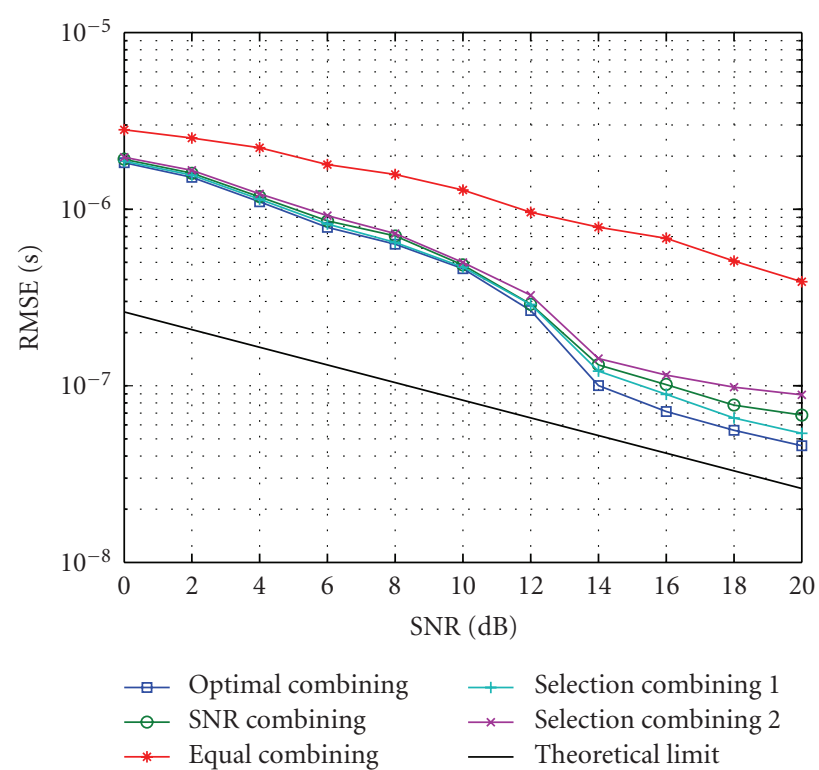

FIGURE 4: RMSE versus SNR for the proposed algorithms, and the theoretical limit (CRLB). The signal occupies three dispersed bands with bandwidths $B_{1}=200 \mathrm{kHz}, B_{2}=100 \mathrm{kHz}$ and $B_{3}=400 \mathrm{kHz}$.

For all the simulations, the spectral densities of the noise at different branches are assumed to be equal; that is, $\sigma_{i}^{2}=\sigma^{2}$ for $i=1, \ldots, K$. In addition, the SNR of the system is defined with respect to the total energy of the signals at different branches; that is, $\mathrm{SNR}=10 \log _{10}\left(\sum_{i=1}^{K} E_{i} /\left(2 \sigma^{2}\right)\right)$.

In assessing the root-mean-squared errors (RMSEs) of the different estimators, a Rayleigh fading channel is assumed. Namely, the channel coefficient $\alpha_{i}=a_{i} \mathrm{e}^{\mathrm{j} \phi_{i}}$ in (1) is modeled as $a_{i}$ being a Rayleigh distributed random variable and $\phi_{i}$ being uniformly distributed in $[0,2 \pi)$. Also, the same average power is assumed for all the bands; namely, $\mathrm{E}\left\{\left|\alpha_{i}\right|^{2}\right\}=1$ is used. The time-delay $\tau$ in (1) is uniformly distributed over the observation interval. In addition, it is assumed that there is no CFO in the system.

First, the performance of the proposed estimators is evaluated with respect to the SNR for a system with $K=3$, $B_{1}=200 \mathrm{kHz}, B_{2}=100 \mathrm{kHz}$, and $B_{3}=400 \mathrm{kHz}$. The results in Figure 4 indicate that the optimal combining technique has the best performance as expected from the theoretical analysis, and SC-1, which estimates the delay according to (20), has performance close to that of the optimal combining technique. The SNR combining and SC-2 techniques have worse performance than that of the optimal and SC1 techniques, respectively. In addition, SC-1 has better performance than that of the SNR combining technique in this scenario, which indicates that selecting the delay estimate corresponding to the largest $\widetilde{E}_{i} \hat{a}_{i}^{2} / \sigma_{i}^{2}$ value is closer to optimal than combining the delay estimates of the different branches according to the SNR combining criterion in (18) for the considered scenario. The main reason for this is related to the large variability of the channel amplitudes due to the nature of the Rayleigh distribution. Since the channel amplitude levels are expected to be quite different for most 


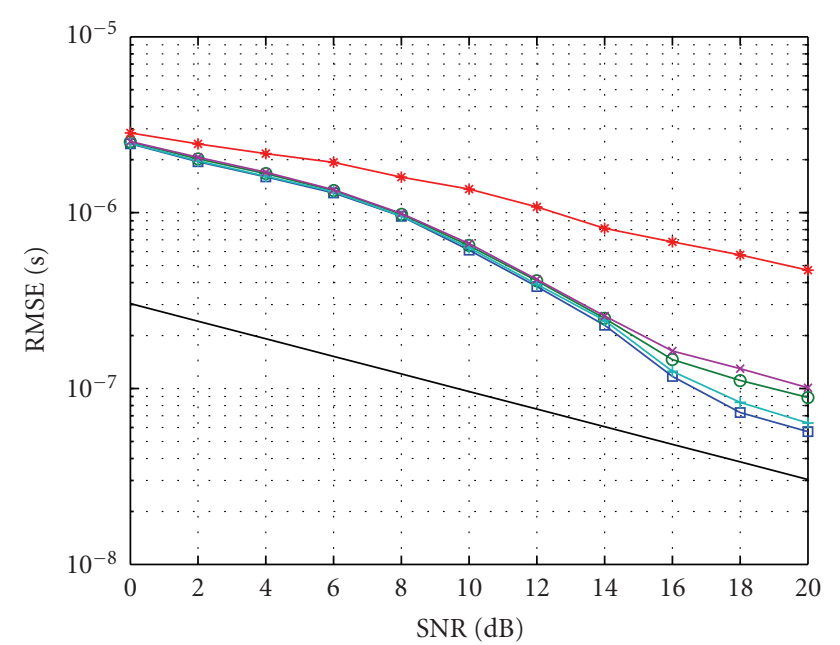

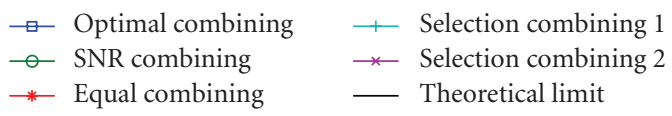

FIGURE 5: RMSE versus SNR for the proposed algorithms, and the theoretical limit (CRLB). The signal occupies two dispersed bands with bandwidths $B_{1}=100 \mathrm{kHz}$ and $B_{2}=400 \mathrm{kHz}$.

of the time, using the delay estimate of the best one yields a more reliable estimate than combining the delay estimates according to the suboptimal SNR combining technique (since the signal bandwidths are different, the SNR combining technique is suboptimal as studied in Section 5). Regarding the equal combining technique, it has significantly worse performance than the others, since it combines all the delay estimates equally. Since the delay estimates of some branches can have very large errors due to fading, the RMSEs of the equal combining technique become significantly larger. For example, when converted to distance estimates, an RMSE of about 120 meters is achieved by this technique, whereas the optimal combining technique results in an RMSE of less than 15 meters. Finally, it is observed that the performance of the optimal combining technique gets very close to the CRLB at high SNRs, which is expected from the asymptotic arguments in Section 5.

Next, similar performance comparisons are performed for a signal with $K=2, B_{1}=100 \mathrm{kHz}$, and $B_{2}=400 \mathrm{kHz}$, as shown in Figure 5. Again similar observations as for Figure 4 are made. In addition, since there are only two bands $(K=2)$ and the signal bandwidths are quite different, the selection combining techniques, SC-1 and SC-2, get very close to the optimal combining and the SNR combining techniques, respectively.

In addition, the equivalence of the optimal combining and the SNR combining techniques and that of SC- 1 and SC-2 are illustrated in Figure 6, where $K=2$ and $B_{1}=$ $B_{2}=400 \mathrm{kHz}$ are used. In other words, the signal consists of two dispersed bands with $400 \mathrm{kHz}$ bandwidths, and in each band, the same signal described by (35) is used. Therefore, $\widetilde{E}_{1} / E_{1}=\widetilde{E}_{2} / E_{2}$ is satisfied, which results in the equivalence of the optimal combining and the SNR combining techniques, as well as that of the SC-1 and SC- 2 techniques, as discussed

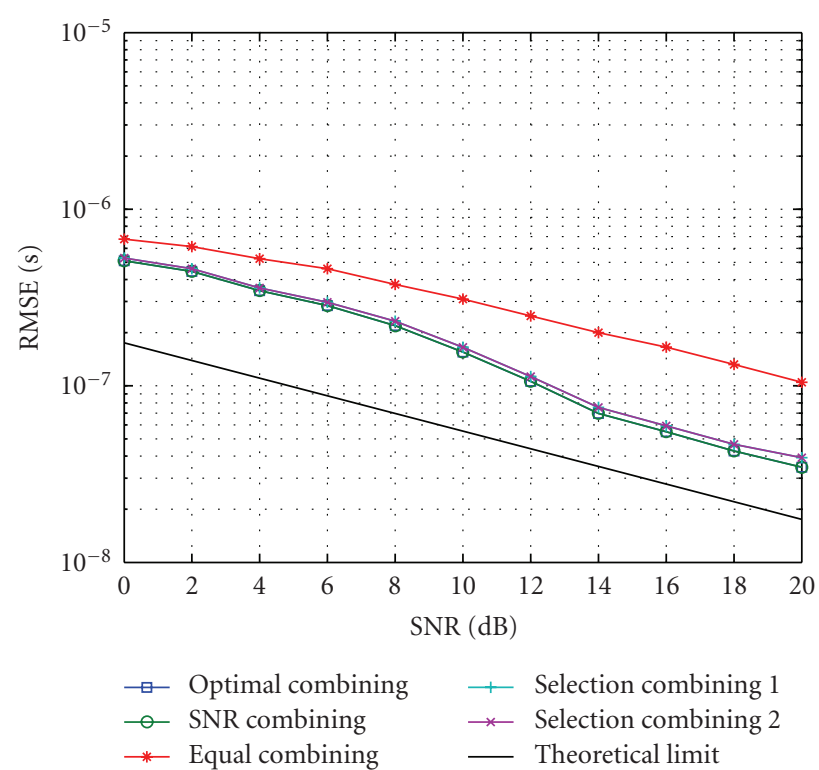

FIGURE 6: RMSE versus SNR for the proposed algorithms, and the theoretical limit (CRLB). The signal occupies two dispersed bands with equal bandwidths of $400 \mathrm{kHz}$.

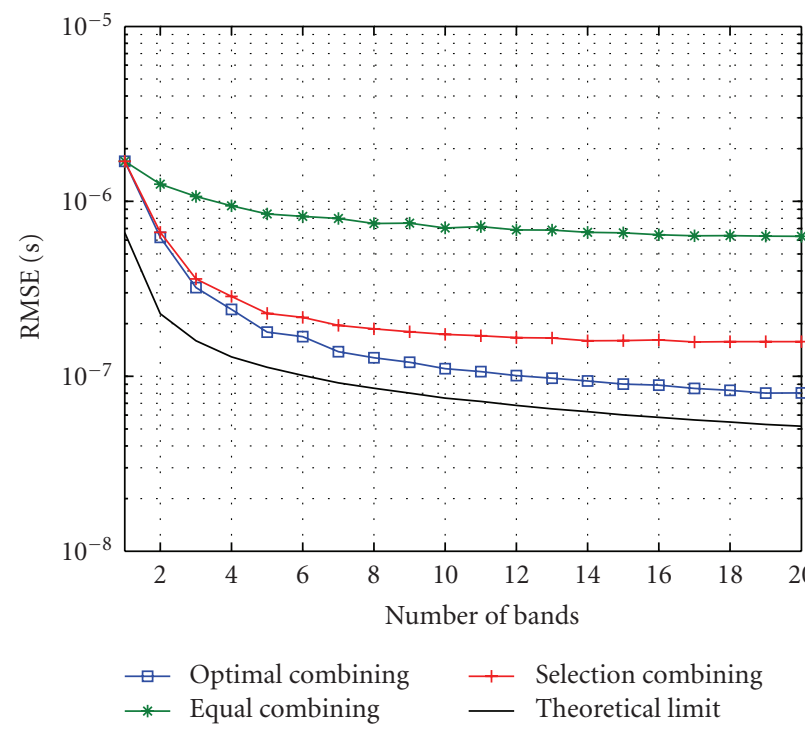

FIgure 7: RMSE versus the number of bands for the proposed algorithms, and the theoretical limit (CRLB). Each band occupies $100 \mathrm{kHz}$, and $\sigma_{i}^{2}=0.1$ for all $i$.

in Section 4. Also, since there are only two bands $(K=2)$, the selection combining techniques get very close to the optimal combining and the SNR combining techniques.

In Figure 7, the RMSEs of the proposed estimators are plotted against the number of bands, where each band is assumed to have $100 \mathrm{kHz}$ bandwidth. The spectral densities are set to $\sigma_{i}^{2}=\sigma^{2}=0.1$ for all $i$. Since the same signals are used in each band, the optimal combining and the SNR combining techniques become identical; hence, only one of them is marked in the figure. Similarly, since SC-1 and SC-2 


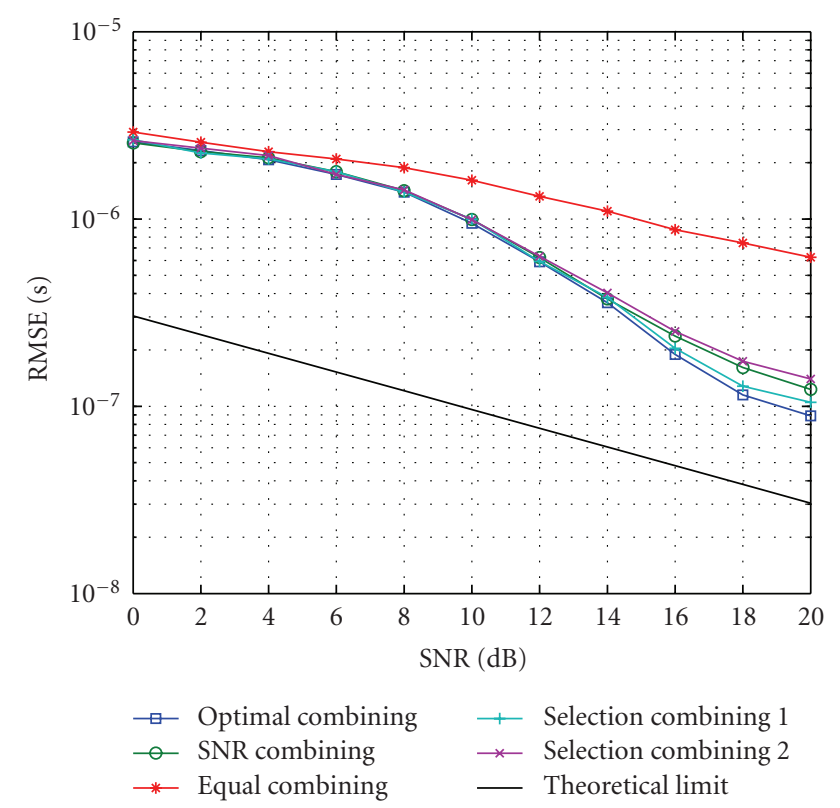

FIGURE 8: RMSE versus SNR for the proposed algorithms, and the theoretical limit (CRLB) in the presence of CFO. The signal occupies two dispersed bands with bandwidths $B_{1}=100 \mathrm{kHz}$ and $B_{2}=400 \mathrm{kHz}$.

are identical in this scenario, they are referred to as "selection combining" in the figure. It is observed from Figure 7 that the optimal combining has better performance than the selection combining and the equal combining techniques. In addition, as the number of bands increases, the amount of reduction in the RMSE per additional band decreases (i.e., diminishing return). In fact, the selection combining technique seems to converge to an almost constant value for large numbers of bands. This is intuitive since the selection combining technique always uses the estimate from one of the branches; hence, in the presence of a sufficiently large number of bands, additional bands do not cause a significant increase in the diversity. On the other hand, the optimal combining technique has a slope that is quite similar to that of the CRLB; that is, it makes an efficient use of the frequency diversity.

Finally, the performance of the proposed algorithms is investigated in the presence of CFO in Figure 8. The CFOs at different branches are modeled by independent uniform random variables over $[-100,100] \mathrm{Hz}$, and the RMSEs are obtained for the system parameters that are considered for Figure 5. Again similar observations as for Figures 4 and 5 are made. In addition, the comparison of Figures 5 and 8 reveals that the RMSE values slightly increase in the presence of CFOs, although the theoretical limit stays the same [23].

\section{Concluding Remarks}

Time-delay estimation for dispersed spectrum cognitive radio systems has been studied. After the investigation of the ML estimator and the CRLBs, a two-step approach has been proposed to obtain accurate time-delay estimates with reasonable computational complexity. In the first step of the proposed approach, an ML estimator is used at each branch of the receiver in order to estimate the unknown parameters of the received signal at that branch. Then, in the second step, a number of diversity combining approaches have been studied. In the optimal combining technique, both the SNRs and the bandwidths of the signals at different branches are considered to obtain the time-delay estimate; whereas the SNR combining technique obtains the timedelay estimate according to the estimated SNR values only. In addition, two selection combining techniques, as well as the equal combining technique, have been investigated. It has been shown that the optimal combining technique can approximate the CRLB at high SNRs; whereas the equal combining technique has the worst performance since it does not make use of any information about signal bandwidths and/or the SNRs. Simulation results have been presented to verify the theoretical analysis.

\section{Acknowledgments}

F. Kocak and S. Gezici wish to acknowledge the activity of the Network of Excellence in Wireless COMmunications NEWCOM++ of the European Commission (Contract no. 216715) that motivated this work. This work was supported in part by the WiMAGIC project of the EC Seventh Framework Programme (FP7) under Grant agreement no. 215167 , and in part by the U. S. National Science Foundation under CNS-09-05398. H. Celebi and K. A. Qaraqe wish to acknowledge the support of Qatar National Funds for Research (QNRF) and Qatar Telecom (Qtel) during this work.

\section{References}

[1] J. Mitola III and G. Q. Maguire Jr., "Cognitive radio: making software radios more personal," IEEE Personal Communications, vol. 6, no. 4, pp. 13-18, 1999.

[2] S. Haykin, "Cognitive radio: brain-empowered wireless communications," IEEE Journal on Selected Areas in Communications, vol. 23, no. 2, pp. 201-220, 2005.

[3] Z. Quan, S. Cui, H. V. Poor, and A. H. Sayed, "Collaborative wideband sensing for cognitive radios," IEEE Signal Processing Magazine, vol. 25, no. 6, pp. 60-73, 2008.

[4] Q. Zhao and B. M. Sadler, "A survey of dynamic spectrum access," IEEE Signal Processing Magazine, vol. 24, no. 3, pp. 7989, 2007.

[5] N. Devroye, M. Vu, and V. Tarokh, "Cognitive radio networks," IEEE Signal Processing Magazine, vol. 25, no. 6, pp. 1223, 2008.

[6] G. Scutari, D. P. Palomar, and S. Barbarossa, "Cognitive MIMO radio," IEEE Signal Processing Magazine, vol. 25, no. 6, pp. 46-59, 2008.

[7] I. Budiarjo, H. Nikookar, and L. P. Ligthart, "Cognitive radio modulation techniques," IEEE Signal Processing Magazine, vol. 25, no. 6, pp. 24-34, 2008.

[8] H. Arslan, Ed., Cognitive Radio, Software Defined Radio, and Adaptive Wireless Systems, Springer, London, UK, 2007. 
[9] J. O. Neel, Analysis and design of cognitive radio networks and distributed radio resource management algorithms, Ph.D. dissertation, Virginia Polytechnic Institute and State University, Blacksburg, Va, USA, 2006.

[10] H. Celebi and H. Arslan, "Enabling location and environment awareness in cognitive radios," Computer Communications, vol. 31, no. 6, pp. 1114-1125, 2008.

[11] Federal Communications Commission (FCC), "Facilitating opportunities for flexible, efficient, and reliable spectrum use employing cognitive radio technologies," ET Docket 03-108, 2005.

[12] M. Wellens, J. Wu, and P. Mahonen, "Evaluation of spectrum occupancy in indoor and outdoor scenario in the context of cognitive radio," in Proceedings of the 2nd International Conference on Cognitive Radio Oriented Wireless Networks and Communications (CrownCom '07), pp. 420-427, Orlando, Fla, USA, August 2007.

[13] S. Haykin, "Cognitive radar: a way of the future," IEEE Signal Processing Magazine, vol. 23, no. 1, pp. 30-40, 2006.

[14] Y. Zhao, J. H. Reed, S. Mao, and K. K. Bae, "Overhead analysis for radio environment mapenabled cognitive radio networks," in Proceedings of the 1st IEEE Workshop on Networking Technologies for Software Defined Radio Networks (SDR '06), pp. 18-25, Reston, Va, USA, September 2006.

[15] H. Celebi and H. Arslan, "Utilization of location information in cognitive wireless networks," IEEE Wireless Communications, vol. 14, no. 4, pp. 6-13, 2007.

[16] S. Yarkan and H. Arslan, "Exploiting location awareness toward improved wireless system design in cognitive radio," IEEE Communications Magazine, vol. 46, no. 1, pp. 128-136, 2008.

[17] H. Celebi and H. Arslan, "Cognitive positioning systems," IEEE Transactions on Wireless Communications, vol. 6, no. 12, pp. 4475-4483, 2007.

[18] P. Mahonen, M. Petrova, and J. Riihijarvi, "Applications of topology information for cognitive radios and networks," in Proceedings of the 2nd IEEE International Symposium on New Frontiers in Dynamic Spectrum Access Networks (DySpan '07), pp. 103-114, Dublin, Ireland, April 2007.

[19] H. Celebi and H. Arslan, "Adaptive positioning systems for cognitive radios," in Proceedings of the 2nd IEEE International Symposium on New Frontiers in Dynamic Spectrum Access Networks (DySpan '07), pp. 78-84, Dublin, Ireland, April 2007.

[20] H. Celebi and H. Arslan, "Utilization of location information in cognitive wireless networks," IEEE Wireless Communications, vol. 14, no. 4, pp. 6-13, 2007.

[21] S. Gezici, "A survey on wireless position estimation," Wireless Personal Communications, vol. 44, no. 3, pp. 263-282, 2008.

[22] S. Gezici, Z. Tian, G. B. Giannakis, et al., "Localization via ultra-wideband radios: a look at positioning aspects of future sensor networks," IEEE Signal Processing Magazine, vol. 22, no. 4, pp. 70-84, 2005.

[23] S. Gezici, H. Celebi, H. V. Poor, and H. Arslan, "Fundamental limits on time delay estimation in dispersed spectrum cognitive radio systems," IEEE Transactions on Wireless Communications, vol. 8, no. 1, pp. 78-83, 2009.

[24] S. Gezici and Z. Sahinoglu, "Ranging in a single-input multiple-output (SIMO) system," IEEE Communications Letters, vol. 12, no. 3, pp. 197-199, 2008.

[25] C. Williams, S. McLaughlin, and M. A. Beach, "Exploiting multiple antennas for synchronization," IEEE Transactions on Vehicular Technology, vol. 58, no. 2, pp. 773-787, 2009.
[26] T. A. Weiss and F. K. Jondral, "Spectrum pooling: an innovative strategy for the enhancement of spectrum efficiency," IEEE Communications Magazine, vol. 42, no. 3, pp. S8-S14, 2004.

[27] S. Brandes, I. Cosovic, and M. Schnell, "Reduction of out-ofband radiation in OFDM systems by insertion of cancellation carriers," IEEE Communications Letters, vol. 10, no. 6, pp. 420422, 2006.

[28] H. A. Mahmoud and H. Arslan, "Sidelobe suppression in OFDM-based spectrum sharing systems using adaptive symbol transition," IEEE Communications Letters, vol. 12, no. 2, pp. 133-135, 2008.

[29] Z. Sahinoglu, S. Gezici, and I. Guvenc, Ultra-Wideband Positioning Systems: Theoretical Limits, Ranging Algorithms, and Protocols, Cambridge University Press, Cambridge, UK, 2008.

[30] H. V. Poor, An Introduction to Signal Detection and Estimation, Springer, New York, NY, USA, 1994.

[31] S. Gezici, Z. Sahinoglu, A. F. Molisch, H. Kobayashi, and H. V. Poor, "Two-step time of arrival estimation for pulse-based ultra-wideband systems," EURASIP Journal on Advances in Signal Processing, vol. 2008, Article ID 529134, 11 pages, 2008.

[32] T.-H. Kim and I.-C. Park, "Two-step approach for coarse time synchronization and frequency offset estimation for IEEE 802.16D systems," in Proceedings of IEEE Workshop on Signal Processing Systems (SiPS '07), pp. 193-198, Shanghai, China, October 2007.

[33] A. Goldsmith, Wireless Communications, Cambridge University Press, Cambridge, UK, 2005. 